\title{
FINGER-KNUCKLE-PRINT RECOGNITION SYSTEM BASED ON FEATURES- LEVEL FUSION OF REAL AND IMAGINARY IMAGES
}

\author{
Abdelouahab Attia ${ }^{1}$, Abdelouahab Moussaoui ${ }^{2}$, Mourad Chaa ${ }^{3}$ and Youssef Chahir ${ }^{4}$ \\ ${ }^{1}$ Department of Computer Science, Mohamed El Bachir El Ibrahimi University of Bordj Bou Arreridj, Algeria \\ ${ }^{2}$ Department of Computer Science, Ferhat Abbas University, Algeria \\ ${ }^{3}$ Department of New Technologies of Information and Communication, Ouargla University, Algeria \\ ${ }^{4}$ Department of Computer Science, University of Caen Lower, France
}

\begin{abstract}
In this paper, a new method based on Log Gabor- TPLBP (LGTPLBP) has been proposed. However the Three Patch Local Binary Patterns (TPLBP) technique used in face recognition has been applied in Finger-Knuckle-Print (FKP) recognition. The 1D-Log Gabor filter has been used to extract the real and the imaginary images from each of the Region of Interest (ROI) of FKP images. Then the TPLBP descriptor on both images has been applied to extract the feature vectors of the real image and the imaginary image respectively. These feature vectors have been jointed to form a large feature vector for each image FKP. After that, the obtained feature vectors of all images are processed directly with a dimensionality reduction algorithm, using linear discriminant analysis (LDA). Finally, the cosine Mahalanobis distance (MAH) has been used for matching stage. To evaluate the effectiveness of the proposed system several experiments have been carried out. The Hong Kong Polytechnic University (PolyU) FKP database has been used during all of the tests. Experimental results show that the introduced system achieves better results than other stateof-the-art systems for both verification and identification.
\end{abstract}

Keywords:

Biometric Systems, Three Patch Local Binary Patterns, 1D Log Gabor filter, Finger Knuckle Print

\section{INTRODUCTION}

The Biometric systems are being generally developed and deployed to improve security to users. Biometric authentication or identification is used to identify a person based on their anatomical or behavioural characteristics such as fingerprint [1] [2] [3], palmprint [4] [5], face [6], iris [7] [8], footprint [9] and FKP [10] [11], etc. FKP is a new type of biometric systems that recognizes a person based on the both knuckle lines and the textures existing in the outer finger surface. These information's (line structures and finger textures) be able to discriminate different individuals, because it is relatively stable and remain unchanged during the life of a person [10] [11] [12]. FKP modality can be by far used by biometric systems because of some advantages. First, data acquisition is relatively simple and reasonable via commercial low-resolution cameras. Second, FKPbased access systems are very adequate for indoor and outdoor usage, and can used even in extreme weather and illumination conditions. Third, FKP features of adults are not disposed to major changes they are more stable over time. Finally, FKP-based biometric information is very reliable and it can be successfully used for recognizing people among several populations [13]. Mainly FKP recognition divided into two tasks: (i) FKP identification and (ii) FKP verification. However, the main goal of FKP identification is to put a given FKP test into one of several predefined sets in a database, whereas FKP verification process is to determine if two FKP images belongs to the same person. In general, the FKP verification task is more difficult than FKP identification because a global threshold have been required to make a decision. FKP recognition has been played a major key in many security fields as well as e-commerce applications, such as biometric access control system, video surveillance, airport checking, computer or mobile devices systems [14][15]. In the literature, many algorithms for FKP recognition have been proposed and investigated. Zhang et al. [10] have proposed a system for authentication of the persons using FKP. This method combines the orientation and the magnitude information extracted by Gabor filter. Guangwei et al. [16] present another approach to personal authentication using a novel multiple orientation coding scheme and texture information that permit to improving the FKP verification accuracy. Shariatmadar and Faez [17] have introduced a system for personal recognition using FKP which combines the extracted features of intensity and Gabor images of each FKP. Zichao et al. [18] have proposed a feature extraction method employing steerable filters which can extract local orientation from FKP images. Tarhouni et al. [19] present an approach to personal recognition using FKP. In their method, the Multi-scale Shift Local Binary Pattern descriptor has been used to define the feature set in the first recognition phase. After that, PCA is used to reduce the dimensionality of feature set and the random subspace is constructed for the most suitable principal subspace. Finally, LDA on this random subspace is used to obtain a final decision. Zeinali et al. [20] have used a system for recognition FKP using Directional Filter Bank (DFB) for feature extraction. Also LDA are used to reduce the dimensionality of the large feature vector. Chaa et al. [21] have merges two types of the histograms of oriented gradients (HOG) based features extracted from reflectance and illumination images FKP. However the authors have employed the Adaptive Single Scale Retinex (ASSR) algorithm in extracting the illumination and the reflectance images from each FKP image. Then HOG descriptor has been applied on both extracted images. These feature vectors have been concatenated to get a large feature vector. Then subspace projection LDA.

In this work a novel approach has been introduced based on Features-Level Fusion of Real and Imaginary Images of FKP image. Firstly, 1D log Gabor filter has been employed to generate the real and the imaginary images from each FKP ROI image (see Fig.1). Then the local binary pattern (LBP) has been used to extract the uniform patterns that contain more useful and selective information in the image. This feature makes the LBP to be suitable for classifying the local spatial structure of an image. In TPLBP a novel patch is employed to ameliorate the performance of the successful Local Binary Pattern (LBP) descriptor. After that, the TPLBP descriptor has been applied on both images (real and imaginary) for extract the feature vectors. Then, these features vectors are concatenated to form a large feature vector for each 
user. Dimensionality reduction of large feature vectors of all users is an important task before classifying the FKP images. Dimensionality reduction is the transformation of highdimensional data onto a lower-dimensional space representation. However, in this work the dimensionality reduction method LDA has been used for FKP recognition and will be presented in detail in this paper. The goal of LDA is to find the global discriminate information for classification based on maximizing the ratio between interclass and intra class scatters [22].

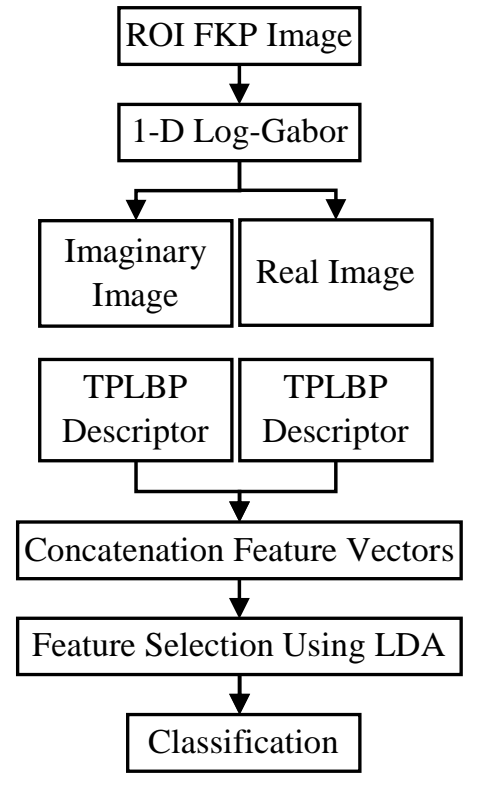

Fig.1. Diagram of the proposed system

The large feature vectors of all users are then subjected to subspace projection. Finally, the cosine Mahalanobis distance (MAH) has been used for matching stage. Finally, to evaluate the effectiveness of the proposed system several experiments have been carried out. The (PolyU) FKP database has been used during all of the tests. This database was collected by Hong Kong Polytechnic University. Experimental results show that the introduced system achieves good results than others systems given in state-of-the-art. The rest of this paper is organized as follows: section 2, describe the proposed FKP recognition system. Section 3 presents the experimental results. Finally, the conclusions and future work are given in the last section

\section{PROPOSED METHOD}

The proposed personal recognition system using FKP performed in four principal stages. The first stage consists in extracting feature vectors of real and imaginary images using Log-Gabor filter and joint these feature vectors. After that, Dimensionality reduction (LDA) done in the second stage. Then, matching and normalized scores realized on third stage. The final stage is the evaluation performances of classifier and decision making. The Fig. 1 clearly illustrates the diagram of the introduced method also the details of these stages are described in the following subsections.

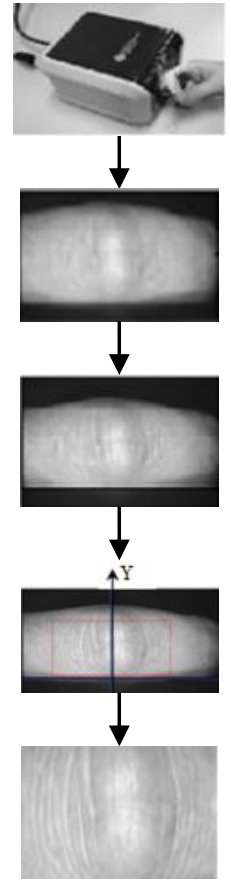

Fig.2. Steps of extraction of FKP ROI image

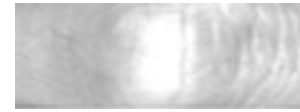

(a)

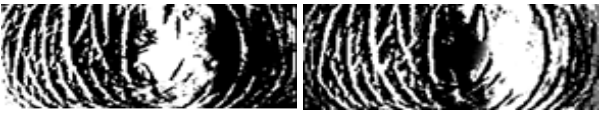

(b) (c)
Fig.3. (a) FKP image (b) Real image (c) Imaginary image

\subsection{THE DATABASE}

In this paper, the FKP images obtained from Hong Kong polytechnic university (PolyU) database [23] has been used to evaluate the proposed method. This database FKP contains 7920 images collected from 165 persons including 125 males and 40 females. Among them, 143 subjects are 20 30 years old and the others are 30 50 years old. Each person provides 48 different FKP images. However, all persons in this database provide 12 images of Left Index Fingers (LIF) and 12 images of Left Middle Fingers (LMF), 12 images of Right Index Fingers (RIF) and 12 images of Right Middle Fingers (RMF). The total number of images RMF or RIF or LIF or LMF is 1980 images provided by 165 persons

\subsection{ROI EXTRACTION}

The extraction the region of interest ROI for FKP [10] is as follows: First, Gaussian smoothing operation have been applied to the original image. Then make down-sample the smoothed image to $150 \mathrm{dpi}$. Second, the X-axis of the coordinate system fixed from the bottom boundary of the finger has been determinate. Also the bottom boundary of the finger can be easily extracted by a canny edge detector. Third, the Y-axis of the coordinate system has been selected by applying a canny edge detector on the cropped sub-image that extracted from the image original base on $\mathrm{X}$-axis, then the convex direction coding scheme has been found. Finally, the ROI system has been extracted, where the rectangle indicates the area of the ROI that will be extracted as shown in Fig. 2. 


\subsection{FEATURE EXTRACTION}

\subsubsection{Log Gabor Filter:}

Gabor filter have been applied as feature extraction method in domain of image analysis and computer vision. But, this filter has some limitations such as in which the even symmetric filter will have a DC component whenever the bandwidth is larger than one octave. Also Gabor filters are not optimal if one is seeking broad spectral information with maximal spatial localization. To overcome these weaknesses, a Log-Gabor filter proposed by Field [24] has been used to eliminate the DC components, thus this filter allowed to produce zero DC components for any band width. LogGabor filter can be constructed with arbitrary bandwidth which can be optimized to produce a filter with minimal spatial extent. The frequency response of a Log-Gabor filter is given by,

$$
G(f)=\exp \left[\frac{-\left(\log \left(\frac{f}{f_{0}}\right)^{2}\right)}{2\left(\log \left(\frac{\sigma}{f_{0}}\right)^{2}\right)}\right]
$$

where, $f_{0}$ represents the centre frequency and $\sigma$ gives the bandwidth of filter. The parameters of Log-Gabor filter were empirically selected as $f_{0}=1 / 2$ and $\sigma=0.0556$ [10]. Each row of the FKP ROI image $(110 * 220$ pixels $)$ is filtered using $1 \mathrm{D}-\mathrm{Log}$ Gabor filter. The result will be $110 \times 220$ arrays of complex numbers. The real and imaginary parts of this array are computed as shown in Fig.3.

\subsubsection{Three-Patch Local Binary Pattern Codes:}

The Three-Patch Local Binary Pattern (TPLBP) is another approach for describing patch-based LBP. The TPLBP code, proposed by Wolf et al. [25] is computed by comparing the values of three patches in order to produce a single bit value in the code assigned to the pixel. The Fig. 4 shows, each pixel of an image is labeled with a TPLBP code that given formally as follows:

$$
\operatorname{TPLBP}_{R, S, W, \alpha}(P)=\sum_{i=0}^{S-1} f\left(d\left(C_{i}, C_{P}\right)-d\left(C_{i+\alpha \bmod s}, C_{P}\right)\right) 2^{i}
$$

In this code, a window of size $w \times w$ has been centered on the pixel. $S$ stand for the total number of windows additional patches distributed uniformly in a ring of radius $r$. we take pairs of patches, $\alpha$ patches apart along the circle, and compare their values with those of the central patch. Final code has $S$ bits per pixel. The parameter $C_{p}$ refers to the central patch where $C_{i}$ and $C_{i}+\alpha \bmod$ $S$ are two patches along the ring. The function $d$ stands for distance function between two patches and $f$ is a threshold function given by,

$$
f(x)=\left\{\begin{array}{lll}
1 & \text { if } & x \geq \tau \\
0 & f & x<\tau
\end{array}\right.
$$

The value $\tau$ is set slightly greater than zero to provide some stability in uniform regions of each real or imaginary image were divided into a grid of none-overlapping regions $(26 \times 26$ grids $)$ and a histogram measuring the frequency of each binary code is calculated for each such region. Each one of these histograms is normalized to unit length, their values truncated at 0.2 , and then once again normalized to unit length. An image is represented by these histograms concatenated to a single vector [25]. After generation the feature vector of real image and the feature vector of imaginary image for each user, it is possible to concatenate these features vectors into one vector. This vector called Large Feature Vector.

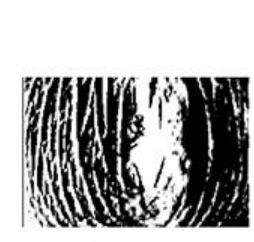

(a)

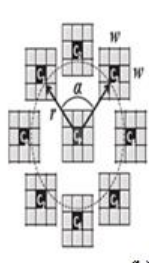

(b)

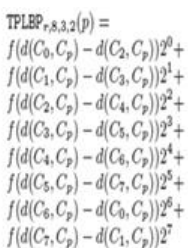
$f\left(d\left(C_{7}, C_{p}\right)-d\left(C_{1}, C_{2}\right)\right) 2^{2}$

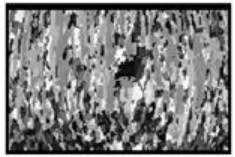

Fig.4. (a) Real FKP image (b) TPLBP code with $S=8, w=3, r$ $=2$ and $\alpha=2$ (c) Code image produced from the real FKP image

\subsubsection{Dimensionality Reduction by LDA:}

LDA is standard technique used in statistical pattern recognition and signal processing for data reduction. The objective of dimensionality reduction is to project the data on a lower dimensional space, which offers computational benefits obtained by processing a smaller amount of data. Let the matrix training $Y_{T}=\left[X_{1}, X_{2}, \ldots, X_{q}\right]$ where $X_{j}$ is the large feature vector of user $(j)$, with $1 \leq j \leq q$ and each element in the set $Y_{T}, X_{j}$ belongs to one of $N$ classes $C_{1}, C_{2}, \ldots, C_{N}$. The within-class scatter matrix $S_{W}$ and the between-class scatter matrix $S_{B}$ are defined as follows:

$$
\begin{gathered}
S_{B}=\sum_{i=1}^{N} n_{i}\left(\mu_{i}-\mu\right)\left(\mu_{i}-\mu\right)^{T} \\
S_{w}=\sum_{i=1}^{N} \sum_{X_{j} \in C_{i}}\left(X_{j}-\mu_{i}\right)\left(X_{j}-\mu_{i}\right)^{T}
\end{gathered}
$$

where, $n_{i}$ denotes the number of samples in the $i^{\text {th }}$ class, $\mu_{i}$ refers to the mean of training sample belonging to the $i^{\text {th }}$ class and $\mu$ represents the global mean of all training samples. Then one derives the LDA transformation matrix $W$ which maximizes Fisher discriminant criterion:

$$
T(W)=W_{\text {opt }}=\underset{W}{\arg \max } \frac{\left|W^{T} S_{B} W\right|}{W^{T} S_{W} W}=\left[W_{1}, W_{2}, \ldots, W_{d}\right]
$$

where, $T(W)$ is the fisher discriminant criterion, that is maximized where $W$ is built by concatenation of the number $d$ leading Eigenvectors. It has been noted that $W$ is obtained by resolving the following system:

$$
S_{W}^{-1} S_{B} W_{j}=W_{j} \lambda_{j}
$$

where, $j=1,2, \ldots, d$ represent the number of training images, where $j$ is much smaller than the number of pixels in image ROI FKP that often results in the singularity of the SW. To overcome the problem of singular SW in this method a PCA transformation step is performed prior to LDA step to avoid singularity issues due to a small number of training samples. This method has been used efficiently in face recognition [20]. The matrix $Y$ of the large feature vectors is projected into Fisher knuckle space by the Eq.(8):

$$
V=W_{\text {opt }}^{T}(Y-\mu)=\left[V_{1}, V_{2}, \ldots, V_{q}\right]
$$




\subsubsection{Matching Module and Normalization Score:}

In this stage we have use the Mahalanobis distance $\left(M_{a}\right)$. Given two vectors $V_{i}=v_{1}, v_{2}, \ldots, v_{n}$ and $Y_{i}=y_{1}, y_{2}, \ldots, y_{n}$, the Ma distance is obtained by the following relation:

$$
d_{M_{a}}\left(V_{i}, Y_{j}\right)=\left(V_{i}, Y_{j}\right)^{T} C^{-1}\left(V_{i}, Y_{j}\right)
$$

where, $C$ stand for the covariance matrix of the training data. Therefore, prior to finding the decision, a method named MinMax normalization scheme was employed to transform the score vectors computed. Given a set of matching scores $\left\{X_{k}\right\}$, where $K$ $=1,2, \ldots, n$. The normalization scores are taken as:

$$
X_{K}^{\prime}=\frac{X_{K-\min }}{\max -\min }
$$

where $X_{K}^{\prime}$ represents the normalized scores.

\section{EXPERIMENTS AND DISCUSSION}

\subsection{SELECTING THE PARAMETERS OF TPLBP ALGORITHM}

The parameters $s, r, w, \alpha$ and grid cell have a great influence on the performance of introduced system. These steps are described below for LMF modality used for right selection of the best parameters:

Step 1: First vary the number of 'alpha' between: 1 to 6 and keep the other parameters fixed as: $r=2, s=8$, grid cell $=26$ and $w=3$. The lowest EER obtained with alpha $=3$ (see Fig.5(a)).

Step 2: In this the values of alpha is fixed to 3 and the values of $w$ are varying between 1 and 6 , and keep the other parameters fixed as: $r=2, s=8$, grid cell $=26$. The lowest EER obtained with alpha $=3$ and $w=3$ (see Fig.5(b)).

Step 3: Fix the values of alpha to 3 and $w=3$ and varying the values of $r$ between 3 to 8 and keep the other parameters fixed as: $s=8$, grid cell $=26$. The lowest EER obtained with alpha $=3, w=3$ and $r=8$ (see Fig.5(c)).

Step 4: Fix the values of alpha to $3, w=3$ and $r=8$, varying the values of grid cell between: 23 to 28 and keep the other parameters fixed as: $\mathrm{s}=8$. The lowest EER obtained with grid cell $=26$. Finally, the best parameters for LMF modality: $s=8, r=8, w=3, \alpha=3$ and grid cell $=26$ (see Fig.5(d)). To sum it up the steps 1 to 4 have been repeated for selecting the best parameters of other modalities. At the end we get the best parameters for each modality that is described as follows:

- LMF modality: $s=8, r=8, w=3, \alpha=3$ and gridCell $=26$.

- RMF modality: $s=8, r=4, w=3, \alpha=2$ and gridCell $=26$.

- LIF modality: $s=8, r=6, w=3, \alpha=5$ and gridCell $=26$.

- RIF modality: $s=8, r=8, w=3, \alpha=3$ and gridCell $=26$.

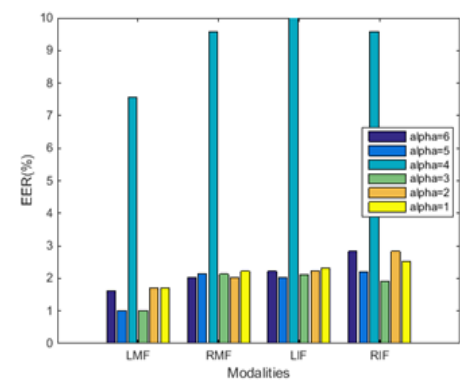

(a)

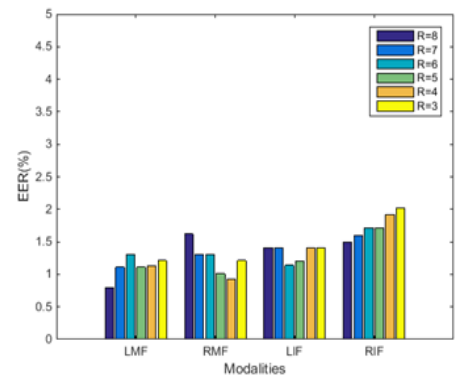

(c)

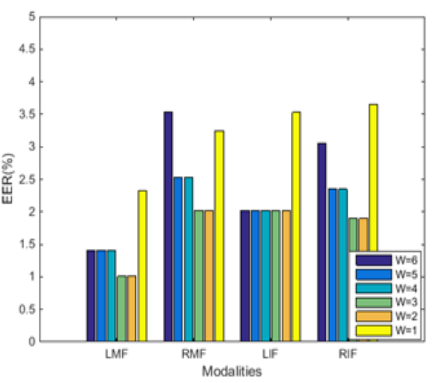

(b)

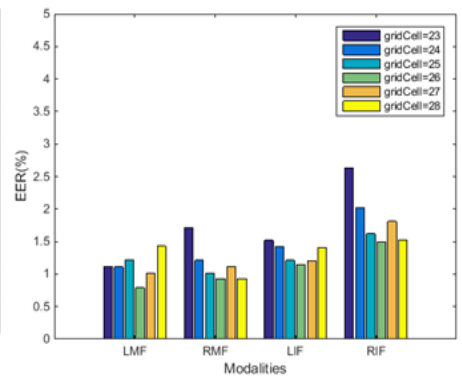

(d)
Fig.5. EER (\%) for the different parameters: (a) alpha (1 to 6), $w$ $=3, r=2$, gridCell $=26$ and $S=8$. (b) $w$ (1 to 6$), r=2$, gridCell $=26$ and $S=8$. (c) $r$ (3 to 8), gridCell $=26$ and $S=8$. (d) gridCell (23 to 28 ) and $S=8$

\subsection{EXPERIMENT 1}

In experiment 1 , only one modality was used separately, (unimodal system). Each modality (LMF or LIF or RMF or RIF) has 12 FKP ROI images for each person collected in two separate sessions (6 images for each session). We use the images of session1 for training for each person (990 images for the training) and the images of session 2 for test (990 images for test). The Table.1 presents the experimental results for both modes (authentication or identification).

Table.1. EER (\%) obtained for the different types fingers and for both modes

\begin{tabular}{|c|c|c|c|c|}
\hline \multicolumn{2}{|c|}{ Mode identification } & \multicolumn{3}{|c|}{ Mode authentication } \\
\hline & ROR (\%) & $\begin{array}{c}\text { EER } \\
\text { (\%) }\end{array}$ & $\begin{array}{c}\text { VR at 1\% } \\
\text { FAR }\end{array}$ & $\begin{array}{c}\text { VR at } \\
\text { 0.1\% FAR }\end{array}$ \\
\hline LMF & $96.16 \%$ & $0.79 \%$ & $99.29 \%$ & $97.58 \%$ \\
\hline RMF & $94.65 \%$ & $0.93 \%$ & $99.19 \%$ & $96.87 \%$ \\
\hline LIF & $95.05 \%$ & $1.14 \%$ & $98.79 \%$ & $96.16 \%$ \\
\hline RIF & $94.34 \%$ & $1.49 \%$ & $98.08 \%$ & $95.56 \%$ \\
\hline
\end{tabular}

For the identification experiments we provide results in the form of recognition rate, using the rank one recognition rate (ROR) is calculated by:

$$
R O R=\frac{N_{1}}{N} \times 100(\%)
$$

where, $N_{i}$ denotes the number of images successfully assigned to the right identity and $N$ stands for the overall number of images trying assign to an identity. The Performance metrics for different finger types are listed in Table.1. It is clear that the highest performance is obtained by using LMF modality, where ROR = 
96.11\%. In the case of using RMF modality, ROR was $94.65 \%$. In the case of LIF modality the system achieve an ROR equal to $95.05 \%$. Finally, RIF modality provide result of $93.34 \%$. The Fig.6 illustrate plot of the CMC curves (Cumulative Match Characteristic) for the different modalities. CMC was used to show the system's performance; however it is a plot of probabilities of correct matches versus the number of best similarity scores. The CMC curve is typically used for identification (one-to-many searches). From the Fig.6, we can see that the accuracy (ROR) is higher when using the proposed method than the accuracy with the use the original image ROI FKP (ROI FKP + TPLBP + LDA + MAH) or real image (real image + TPLBP + LDA + MAH) or Imaginary image (Imaginary image + TPLBP + LDA + MAH). For the authentication experiments we provide results in the form of EER where FAR = FRR for different finger types are listed in Table.1. It is clear that the lowest EER is obtained by LMF modality, in this case the proposed system gives EER $=0.79 \%$. EER $=0.93 \%$ for the RMF modality, this EER was $1.14 \%$ for the LIF and EER $=1.49 \%$ for the RIF modality. To evaluate the performance of verification, the detection error trade-off (DET) curve are used, and the results are shown in Fig.7. From Fig.6, we can see that the lowest EER is obtained when using the proposed method.

The comparison of the EER/ROR (\%) of proposed system unimodal in both authentication and identification modes with the other systems presented in the other papers under the same condition is given in the Table.2. It is clear that the introduced system gives highest ROR and lowest EER excepting method given in reference [26].

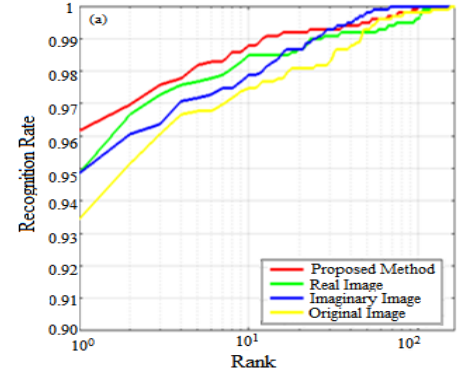

(a)

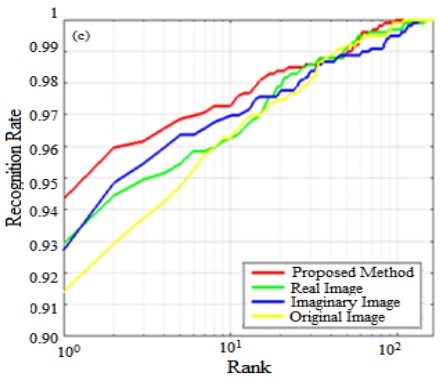

(c)

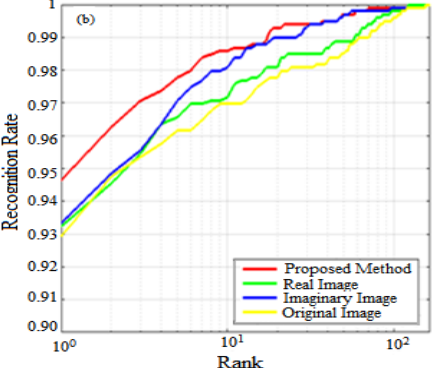

(b)

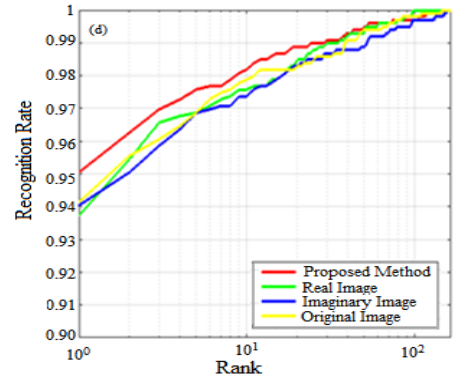

(d)
Fig.6. CMC curves for the different modalities: (a) LMF (b) RMF (c) RIF (d) LIF
Table.2. Comparison of our system unimodal in both modes identification and authentication

\begin{tabular}{|c|c|c|c|c|c|c|c|c|}
\hline & \multicolumn{2}{|c|}{ LMF } & \multicolumn{2}{|c|}{ RMF } & \multicolumn{2}{|c|}{ LIF } & \multicolumn{2}{|c|}{ RIF } \\
\hline & EER & ROR & EER & ROR & EER & ROR & EER & ROR \\
\hline MSLBP [19] & - & $\begin{array}{c}94.70 \\
\%\end{array}$ & - & $\begin{array}{c}94.80 \\
\%\end{array}$ & - & $\begin{array}{c}93.80 \\
\%\end{array}$ & - & $\begin{array}{c}92.20 \\
\%\end{array}$ \\
\hline $\begin{array}{l}\text { Intensity + } \\
\text { Gabor [17] }\end{array}$ & - & $\begin{array}{c}88.59 \\
\%\end{array}$ & - & $\begin{array}{c}88.48 \\
\%\end{array}$ & - & $\begin{array}{c}89.90 \\
\%\end{array}$ & - & $\begin{array}{c}89.49 \\
\%\end{array}$ \\
\hline $\begin{array}{l}\text { DFB + LDA } \\
+ \text { Norm [20] }\end{array}$ & - & $\begin{array}{c}90.30 \\
\%\end{array}$ & - & $\begin{array}{c}89.79 \\
\%\end{array}$ & - & $\begin{array}{c}88.68 \\
\%\end{array}$ & - & $\begin{array}{c}89.79 \\
\%\end{array}$ \\
\hline $\begin{array}{c}\text { ImCompCo } \\
\text { de \& } \\
\text { Magcode } \\
{[10]}\end{array}$ & $\begin{array}{c}1.78 \\
\%\end{array}$ & - & $\begin{array}{c}1.64 \\
\%\end{array}$ & - & $\begin{array}{c}1.73 \\
\%\end{array}$ & - & $\begin{array}{c}1.44 \\
\%\end{array}$ & - \\
\hline $\begin{array}{c}\text { MoriCode \& } \\
\text { MtexCode } \\
{[16]}\end{array}$ & $\begin{array}{c}1.45 \\
\%\end{array}$ & - & $\begin{array}{c}1.06 \\
\%\end{array}$ & - & $\begin{array}{c}1.32 \\
\%\end{array}$ & - & $\begin{array}{c}1.24 \\
\%\end{array}$ & - \\
\hline [26] & $\begin{array}{c}0.38 \\
\%\end{array}$ & $\begin{array}{c}95.54 \\
\%\end{array}$ & $\begin{array}{c}0.35 \\
\%\end{array}$ & $\begin{array}{c}96.72 \\
\%\end{array}$ & $\begin{array}{c}0.54 \\
\%\end{array}$ & $\begin{array}{c}94.33 \\
\%\end{array}$ & $\begin{array}{c}0.78 \\
\%\end{array}$ & $\begin{array}{c}95.93 \\
\%\end{array}$ \\
\hline $\begin{array}{l}\text { Proposed } \\
\text { method }\end{array}$ & $\begin{array}{c}0.79 \\
\%\end{array}$ & $\begin{array}{c}96.16 \\
\%\end{array}$ & $\begin{array}{c}0.93 \\
\%\end{array}$ & $\begin{array}{c}94.65 \\
\%\end{array}$ & $\begin{array}{c}1.14 \\
\%\end{array}$ & $\begin{array}{c}95.05 \\
\%\end{array}$ & $\begin{array}{c}1.49 \\
\%\end{array}$ & $\begin{array}{c}94.34 \\
\%\end{array}$ \\
\hline
\end{tabular}

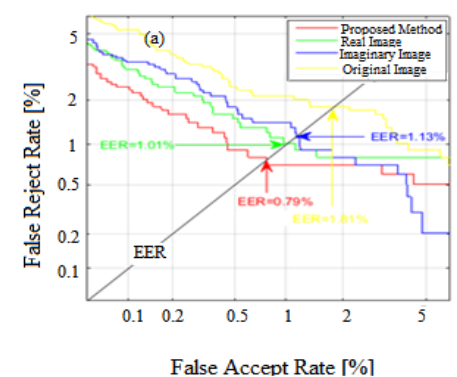

(a)

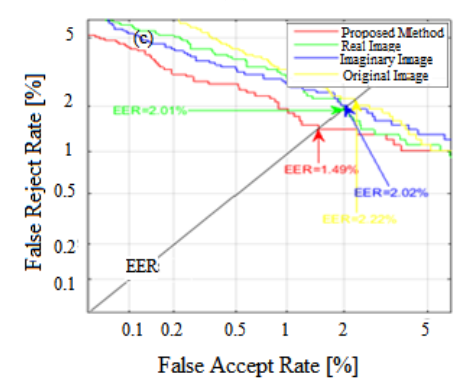

(c)

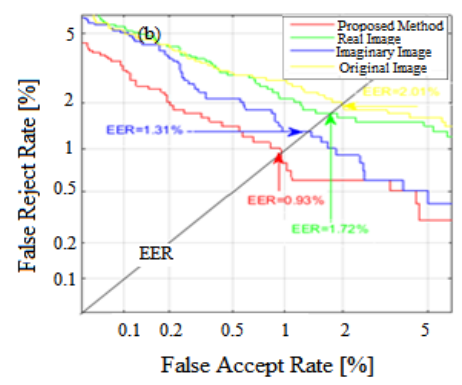

(b)

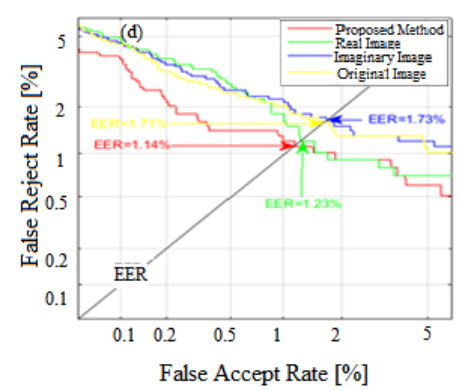

(d)
Fig.7. DET curves for the different modalities: (a) LMF (b) RMF (c) RIF (d) LIF

\subsection{EXPERIMENT 2}

In this experiment, we use two or more fingers types of the same person for increase the performances of introduced system. Thus, we can enhance the recognition performance of the FKP recognition algorithm by using multiple fingers. After the generation of the feature vector for each finger types of the same user (LMF, LIF, RMF and RIF), it is possible to fusion these (two or more vectors) into single vector for each user. We use 6 vectors for training for each person (990 vectors for the training) and 6 vectors for test (990 vectors for test). These vectors of all users are then subjected to subspace projection LDA. The nearest neighbor 
classifier and MAH distance are used for respectively matching and decision stages.

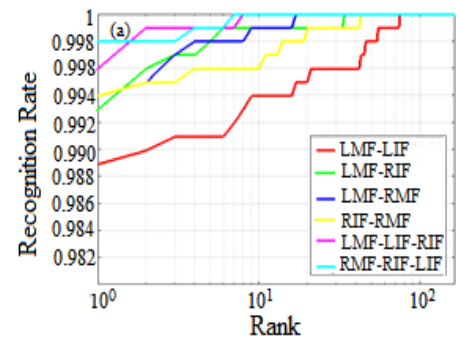

(a)

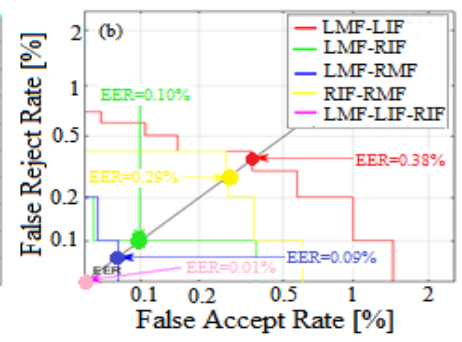

(b)
Fig.8. Curves (a) CMC curve (b) DET for the different combinations

The Table. 3 presents our experimental results for both modes (authentication (EER) or Identification (ROR)). The introduced system multi-modal gives the $\mathrm{ROR}=99.39 \%$ for the combinations (LMF-RMF) and (RIF-RMF). ROR was $99.29 \%$ for the combination (LMF-RIF). This ROR was $99.09 \%$ for the combination (LIF-RIF). Finally, this ROR $=98.89 \%$ for the combination (LMF-LIF). As can be seen, the best result was obtained with the combination of three or four fingers; it can achieve even higher precision, an ROR of $99.80 \%$ for the combinations (RMF-RIF-LIF) and (RMF-RIF-LMF). ROR was $99.60 \%$ for the combination (LMF-LIF-RIF) and was $99.39 \%$ for the combination (LMF-LIF-RMF). ROR was $100.00 \%$ for the combination all fingers. The Table. 3 tabulates the EER (\%) for various combinations possible, the proposed system multi-modal gives the EER $=0.38 \%$ for the combinations (LMF-LIF). EER was $0.10 \%$ for the combinations (LMF-RIF) and (RMF-LIF). This EER was $0.09 \%$ for the combination (LMF-RMF). Finally, this EER $=0.20 \%$ for the combination (LIF-RIF). As can be seen, the lowest EER was obtained with the combination of three or all fingers. Our system can achieve an EER of $0.01 \%$ for the combinations (LMF-LIF-RIF), (LMF-LIF-RMF) and (RMF-RIFLIF). EER was $0.02 \%$ for the combination (RMF-RIF-LMF).
EER was $00.00 \%$ for the combination all fingers. The Fig.8(a) present the plot of the CMC curve for the different combinations. The Fig.8(b) plot the DET curve for the different combination. The comparison of the EER/ROR (\%) of our system multimodal with the EER/ ROR of the other systems presented in the other papers under the same condition is given in the Table.4. It is clear that our system gives the highest ROR and lowest EER excepting method given in [26].

Table.3. EER/ROR (\%) Obtained by the fusion of the different fingers type

\begin{tabular}{|c|c|c|c|c|c|c|}
\hline \multirow{3}{*}{$\begin{array}{c}\text { EER/ROR } \\
(\%) \\
\text { Auth/Ident }\end{array}$} & \multicolumn{6}{|c|}{ Fusion of Two Types Fingers } \\
\hline & \multicolumn{2}{|c|}{ LMF-LIF } & \multicolumn{2}{|c|}{ LMF-RIF } & \multicolumn{2}{|c|}{ LMF-RMF } \\
\hline & EER & ROR & EER & ROR & EER & ROR \\
\hline & $0.40 \%$ & & $0.30 \%$ & & & \\
\hline & $0.40 \%$ & & & & & \\
\hline & $50 \%$ & & & & & \\
\hline & $0.38 \%$ & 98.8 & $0.10 \%$ & & & \\
\hline \multirow{2}{*}{$\begin{array}{c}\text { EER/ROR } \\
(\%) \\
\text { Auth/Ident } \\
\end{array}$} & \multicolumn{2}{|c|}{ RIF-RMF } & \multicolumn{2}{|c|}{ RMF-LIF } & \multicolumn{2}{|c|}{ LIF-RIF } \\
\hline & EER & ROR & EER & ROR & EER & ROR \\
\hline Sum rule & $0.40 \%$ & $98.79 \%$ & $0.30 \%$ & & & \\
\hline & $0.79 \%$ & $96.87 \%$ & $0.72 \%$ & $96.06 \%$ & $0.83 \%$ & $96.26 \%$ \\
\hline Max & $0.39 \%$ & $98.79 \%$ & $0.20 \%$ & & & 98. \\
\hline & $29 \%$ & $99.39 \%$ & $0.10 \%$ & $99.19 \%$ & $0.20 \%$ & $99.09 \%$ \\
\hline \multirow{3}{*}{$\begin{array}{c}\text { EER/ROR } \\
(\%) \\
\text { Auth/Ident }\end{array}$} & \multicolumn{6}{|c|}{ Fusion of Three or all Types Fingers } \\
\hline & \multicolumn{2}{|c|}{ LMF-LIF-RIF } & \multicolumn{2}{|c|}{\begin{tabular}{|c|} 
LMF-LIF- \\
RMF
\end{tabular}} & \multicolumn{2}{|c|}{ RMF-RIF-LMF } \\
\hline & EER & ROR & EER & ROI & EER & ROR \\
\hline & $0.12 \%$ & $99.49 \%$ & $0.05 \%$ & 99.1 & $0.20 \%$ & $99.19 \%$ \\
\hline & $0.61 \%$ & $96.36 \%$ & $1.01 \%$ & $96.67 \%$ & $1.01 \%$ & $94.75 \%$ \\
\hline Max & $0.20 \%$ & $99.49 \%$ & $0.08 \%$ & $99.29 \%$ & $0.19 \%$ & $99.39 \%$ \\
\hline Concatenation & $0.01 \%$ & $99.60 \%$ & $0.01 \%$ & $99.39 \%$ & $0.02 \%$ & $99.80 \%$ \\
\hline
\end{tabular}

Table.4. Comparison of the EER/ROR (\%) of our system multimodal with the EER/ ROR of the other systems

\begin{tabular}{|c|c|c|c|c|c|c|c|c|}
\hline & \multicolumn{2}{|c|}{ LIF-LMF } & \multicolumn{2}{c|}{ LIF-RIF } & \multicolumn{2}{c|}{ LIF-RMF } & \multicolumn{2}{c|}{ LMF-RIF } \\
\cline { 2 - 9 } & EER & ROR & EER & ROR & EER & ROR & EER & ROR \\
\hline Intensity + Gabor [17] & - & $95.56 \%$ & - & $95.25 \%$ & - & $95.05 \%$ & - & $94.65 \%$ \\
\hline DFB + LDA + Norm [20] & - & $95.55 \%$ & - & $97.27 \%$ & - & $96.46 \%$ & - & $97.57 \%$ \\
\hline$[26]$ & 0.125 & $99.09 \%$ & 0.162 & $98.69 \%$ & 0.13 & $98.79 \%$ & 0.143 & $98.79 \%$ \\
\hline$[21]$ & - & $98.69 \%$ & - & $98.08 \%$ & - & $98.59 \%$ & - & $98.69 \%$ \\
\hline Proposed method & $0.40 \%$ & $98.79 \%$ & - & - & - & - & $0.30 \%$ & $98.48 \%$ \\
\hline & LIF-LMF-RIF & LIF-LMF-RMF & LIF-RIF-RMF & Four fingers \\
\cline { 2 - 10 } & EER & ROR & EER & ROR & EER & ROR & EER & ROR \\
\hline Intensity + Gabor [17] & - & $95.66 \%$ & - & $96.06 \%$ & - & $95.86 \%$ & - & $96.56 \%$ \\
\hline DFB + LDA + Norm [20] & - & $98.08 \%$ & - & $98.18 \%$ & - & $98.68 \%$ & - & $99.29 \%$ \\
\hline$[26]$ & $0.048 \%$ & $99.7 \%$ & $0.032 \%$ & $99.6 \%$ & $0.015 \%$ & $99.8 \%$ & $0.0 \%$ & $100 \%$ \\
\hline$[21]$ & - & - & - & - & - & - & 0.00 & $100 \%$ \\
\hline Proposed method & $0.01 \%$ & $99.60 \%$ & $0.01 \%$ & $99.39 \%$ & $0.01 \%$ & $99.80 \%$ & $0.00 \%$ & $100 \%$ \\
\hline
\end{tabular}




\section{CONCLUSION}

In this proposed biometric system, the 1D log Gabor filter algorithm is employed to generate the real and imaginary images respectively from every one of image ROI FKP. The TPLBP descriptor is applied on the both images to extract the feature vectors of the real image and the imaginary image. These feature vectors are jointed to form a large feature vector for each user. Then these feature vectors of all users are subjected to subspace projection LDA. Finally, nearest neighbor classifier with MAH distance is used for matching and decision stages respectively. It has been found that the performance is higher when using the 1Dlog-Gabor filter in our system for extracting the feature vectors by the proposed method than the performance without the use 1Dlog-Gabor filter for extracting the feature vectors. In addition, we demonstrate that the use of multiple modalities makes it possible to significantly improve the performance of the FKP recognition algorithm. The future work will focus on the integration of other biometric traits such as fingerprint or palmprint to get the system performances with a high accuracy by using a large database.

\section{REFERENCES}

[1] D. Maltoni, D. Maio, A.K. Jain and S. Prabhakar, "Handbook of Fingerprint Recognition", Springer, 2003.

[2] Q. Zhao, D. Zhang, L. Zhang and N. Luo, "Adaptive Fingerprint Pore Modeling and Extraction", Pattern Recognition, Vol. 43, No. 8, pp. 2833-2844, 2010.

[3] G. Jaswal, A. Nigam and R. Nath, "DeepKnuckle: Revealing the Human Identity", Multimedia Tools and Applications, Vol. 76, No. 18, pp. 18955-18984, 2017.

[4] W. Jia, D. Huang and D. Zhang, "Palmprint Verification based on Robust Line Orientation Code", Pattern Recognition, Vol. 41, No. 5, pp. 1504-1513, 2008.

[5] Z. Guo, D. Zhang, L. Zhang and W. Zuo, "Palmprint Verification using Binary Orientation Co- Occurrence Vector", Pattern Recognition Letters, Vol. 30, No. 13, pp. 1219-1227, 2009.

[6] W. Zhao, R. Chellappa, P.J. Phillips and A. Rosenfeld, "Face Recognition: A Literature Survey", ACM Computing Surveys, Vol. 35, No. 4, pp. 339-458, 2003.

[7] J. Daugman, "How Iris Recognition Works", IEEE Transactions on Circuits and Systems for Video Technology, Vol. 14, No. 1, pp. 21-30, 2004.

[8] W. Dong, Z. Sun and T. Tan, "Iris Matching based on Personalized Weight Map", IEEE Transactions on Pattern Analysis and Machine Intelligence, Vol. 33, No. 9, pp. 17441757, 2011.

[9] A. Uhl and P. Wild, "Footprint-based Biometric Verification", Journal of Electronic Imaging-Society of Photo-Optical Instrumentation Engineers, Vol. 17, No. 1, pp. 110-116, 2008.

[10] L. Zhang, L. Zhang, D. Zhang and H. Zhu, "Online FingerKnuckle-Print Verification for Personal Authentication", Pattern Recognition, Vol. 43, No. 7, pp. 2560-257, 2010.

[11] A. Kumar and Ch. Ravikanth, "Personal Authentication using Finger Knuckle Surface", IEEE Transactions on Information Forensics and Security, Vol. 4, No. 1, pp. 98-109, 2009.
[12] S. Prabhakar, and A.K. Jain, "Decision-Level Fusion in Fingerprint Verification”, Pattern Recognition, Vol. 35, No. 4, pp. 861-874, 2002.

[13] A. Meraoumia, S. Chitroub and A. Bouridane, "Palmprint and Finger-Knuckle-Print for Efficient Person Recognition based on Log-Gabor Filter Response", Analog Integrated Circuits and Signal Processing, Vol. 69, No. 2, pp.17-27, 2011.

[14] K.A. Toh, X. Jiang and W.Y. Yau, "Exploiting Global and Local Decisions for Multimodal Biometrics Verification", IEEE Transactions on Signal Processing, Supplement on Secure Media, Vol. 52, No. 10, pp. 3059-3072, 2004.

[15] A. Ross, D. Nandakumar, and A.K. Jain, "Handbook of Multibiometrics", Springer, 2006.

[16] G. Gao, J. Yang, J. Qian and L. Zhang, "Integration of Multiple Orientation and Texture Information for FingerKnuckle-Print Verification", Neurocomputing, Vol. 135, pp. 180-191, 2014.

[17] Z.S. Shariatmadar and K. Faez, "An Efficient Method for Finger Knuckle- Print Recognition by using the Information Fusion at Different Levels", Proceedings of IEEE International Conference on Hand-Based Biometrics, pp. 16, 2011.

[18] L. Zichao, K. Wang and W. Zuo, "Finger-Knuckle-Print Recognition using Local Orientation Feature based on Steerable Filter", Proceedings of International Conference on Intelligent Computing, Vol. 304, pp. 224-230, 2012.

[19] W. El-Tarhouni, M.K. Shaikh, L. Boubchir and A. Bouridane, "Multi-Scale Shift Local Binary Pattern BasedDescriptor for Finger-Knuckle-Print Recognition", Proceedings of $26^{\text {th }}$ International Conference on Microelectronics, pp. 408-413, 2014.

[20] B. Zeinali, A. Ayatollah and M. Kakooei, "A Novel Method of Applying Directional Filter Bank for Finger-KnucklePrint Recognition", Proceeding of $22^{\text {nd }}$ Iranian Conference on Electrical Engineering, pp. 500-504, 2014.

[21] M. Chaa, N. Boukezzoula and A. Meraoumia, "FeaturesLevel Fusion of Reflectance and Illumination Images in Finger-Knuckle-Print Identification System", International Journal on Artificial Intelligence Tools, Vol. 27, No. 3, pp. 1-10, 2018.

[22] P.N. Belhumeur, J.P. Hespanha and D.J. Kriegman, "Eigenfaces vs. Fisherfaces: Recognition using Class Specific Linear Projection", IEEE Transactions on Pattern Analysis and Machine Intelligence, Vol. 19, No. 7, pp. 711-720, 1997.

[23] FKP Database, Available at: http://www4.comp.polyu.edu.hk/ biometrics/FKP.htm, Accessed on 2010.

[24] D.J. Fiel, "Relations between the Images and the Response Properties of Cortical Cells", Journal of the Optical Society of America A, Optics and Image Science, Vol. 4, No. 2, pp. 2379-2394, 1987.

[25] L. Wolf, T. Hassner and Y. Taigman, "Descriptor based Methods in Wild.in Real-Life Images", Proceedings of European Conference on Computer Vision, pp. 12-17, 2008

[26] S.Z. Shariatmadar and K. Faez, "Finger-Knuckle-Print Recognition via Encoding Local-Binary-Pattern", Journal of Circuits, Systems and Computers, Vol. 22, No. 6, pp. 1$16,2013$. 